\title{
Las grandes ciudades del Indo
}

\author{
M. ${ }^{a}$ TeResa Román LÓPEZ*
}

\begin{abstract}
RESUMEN
ABSTRACT

La civilización del Indo (alrededor del tercer milenio a.C.), llamada también cultura de Harappa, tenía una superficie más o menos triangular que rondaba el millón trescientos mil kilómetros cuadrados. La mayor parte de los 150 emplazamientos de esta cultura de la Edad del Bronce se hallan en Pakistán. Los centros principales fueron los dos asentamientos de mayor tamaño: Mohenjo-Daro y Harappa. The Indo civilization in the third milenium, called Harappa culture had about 150 deposits in the Bronce Age, actual Pakistan, its main deposits were Mohenho Daro and Harappa. Their constructions, made on boiled and bricks and wood were quite perfect with important hidruilic ingenier technics. Its urban centers had an interestic architecture. Ist decadence in the XVI century was replace for a rural culture.
\end{abstract} La rigurosa funcionalidad de las construcciones, en ladrillos cocidos y madera, la nítida realización de la implantación, la técnica hidráulica de los constructores, evidente en canales, cañerías de alimentación y alcantarillas, dan a esta arquitectura protoindia un carácter racionalista. Los centros urbanos presentan, en posición elevada, la acrópolis, cercada de muros y rica en terrazas, palestras

* Departamento de Filosofía y Filosofía Moral y Política. UNED. 
La segunda zona corresponde al «barrio indio». Aquí, las calles estrechas están llenas de mujeres con coloridos saris y hombres vestidos con dhotis (largos taparrabos) Las numerosas y pequeñas tiendas se prolongan hasta las aceras, donde vendedores ambulantes, barberos y escritores de cartas anuncian en voz alta sus mercancías o servicios.

Fuera de esta prosperidad y bullicio, en los barrios más pobres de las ciudades y en sus proximidades, se hallan los territorios poblados de infraviviendas, cuya población crece todos los años debido a la inmigración procedente de las aldeas, donde, con mucha frecuencia, estos sólo encuentran el paro, la miseria y la degradación social.

\section{LA CIVILIZACIÓN DEL VALLE DEL INDO}

Las colaboraciones de la Royal Asiatic Society y, más adelante, de la Archaelogical Survey para la recuperación del pasado de India han sido inconmensurables. Bajo los auspicios de la segunda se descubrió una civilización prearia (figura 1) que aparece emparentada con la de Mesopota-

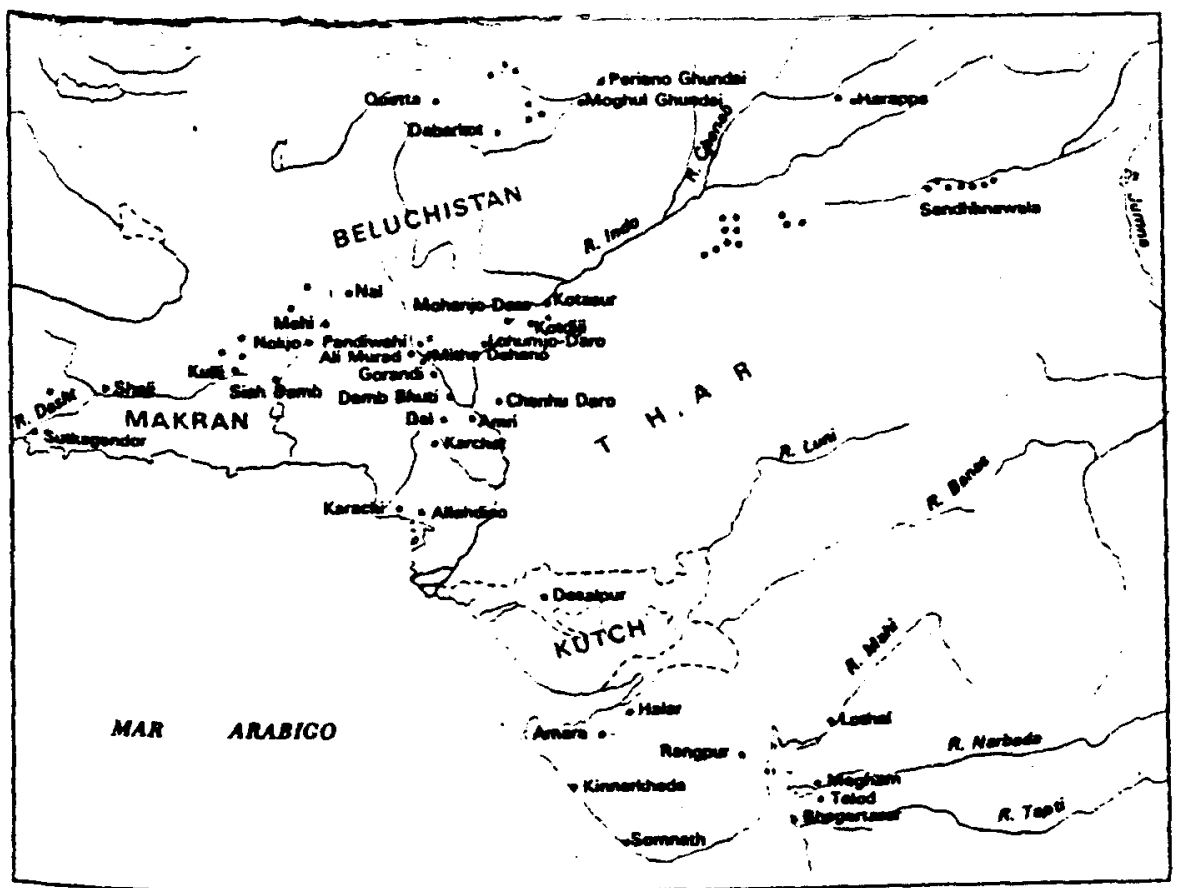

Figura 1. Yacimientos arqueológicos de la India prearia. 
mia, en la cuenca del Indo. Las excavaciones se han verificado en Mohenjo-Daro (distrito de Larkana, provincia de Sindh), y en Harappa (distrito de Montgomery, Punjab).

En el año 1924 Sir John Marshall dio la noticia de dos extraordinarios descubrimientos en Mohenjo-Daro y Harappa (figura 2). Ambos, entre otros hallazgos, hicieron retroceder varios milenios la historia conocida de la India ${ }^{2}$.

Las dos ciudades excavadas hasta ahora no son las únicas, pues se reconoce que debe de haber otras sepultadas en montículos del valle del Indo ${ }^{3}$. Su abundancia confirma la información, acaso algo exagerada

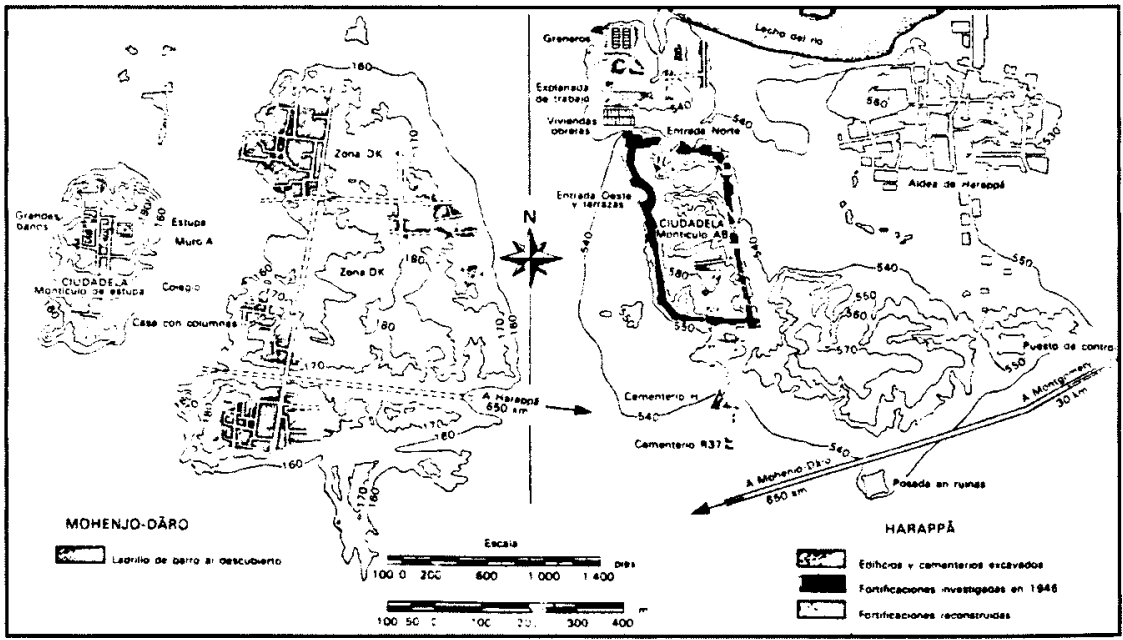

Figura 2. Mohenjo-Daro y Harappa: los planos generales comparados (según Wheeler).

2 Hasta la segunda década del siglo $x x$, fecha en que tuvieron lugar las primeras excavaciones en Mohenjo-Daro y en Harappa, no se sabía nada de la civilización del Indo. De hecho, la segunda se descubrió en el siglo XIX, pero no se reparó en su importancia y fue saquedada para obtener balasto durante la construcción del ferrocarril de Lahore. En 1931 se descubrió otra ciudad en Chanchu-daro, más cerca de la desembocadura del Indo. A partir de esa fecha se han desenterrado nuevos yacimientos, incluidos Kalibangan, en el norte de Rajastán, Lothal y Rojdi, en la zona de Cambai, y Sutkagen Dor, que antaño estuvo en la costa de Makran. También se han emprendido nuevas excavaciones en Mohenjo-Daro y Harappa.

3 Se han hallado vestigios de la misma civilización en lugares tan distantes como Kathiawar en el Oeste y en el distrito de Ambala, en el Punjab, y hay motivos para pensar que se extendía también hasta el valle del Ganges. No se trataba, pues, de la "civilización del valle del Indo", sino de una cultura muy difundida por vastas áreas de la India, especialmente en el Norte. Al parecer se trataba de una civilización muy evolucionada, que debió tardar muchos siglos en llegar al esplendor indicado por estos descubrimientos arqueológicos. Ver E.O, JamEs (dir.), Historia de las religiones, Vergara, Barcelona, 1963, vol. I, pág. 336. 
en el Rig Veda ${ }^{4}$, de las setenta mil fortalezas gobernadas por sesenta jefes.

De los habitantes de estas ciudades del Indo no se sabe mucho. Al contrario de lo ocurrido en Mesopotamia, Egipto y China, aquí no se han encontrado hasta ahora las tumbas de la clase dominante. Los escasos restos humanos hasta ahora publicados de manera satisfactoria desde un punto de vista científico proceden, lamentablemente, del último período de Mohenjo-Daro. Ofrecen una facies antropológica muy compleja, se diría que incluso una mezcla de razas: proto-australoide, mediterránea, alpina, alpino-mongoloide.

Diversos estudios han permitido retrotraer al año 2500 a.C. el origen de estas culturas para las que podemos fijar tres períodos: Amri, Harappa y Jhucar ${ }^{5}$.

La cuitura de $A m r i^{6}$, situada en el valle del Indo, también se la conoce con el nombre de "poblados de colina» (Zhob, Amri, Quetta, Kot Diji, Togau), llegó a su máximo apogeo hacia finales del IV milenio.

Antecede a Mohenjo-Daro y Harappa en la planificación urbana, en las formas cerámicas y en la clase de fortificación. Los estratos más antiguos de la cultura del Indo están quemados, lo que significa una violenta destrucción del asentamiento por las gentes de Harappa en un momento en que las de Kot Diji (a unos cien kilómetros al norte de Haidarabad, en Sindh) habian conseguido un importante desarrollo en la vida social y en determinados aspectos de su cultura material, por ejemplo, la cerámica pintada. De ello se deduce que no constituian repúblicas federadas en permanente armonia, pues la presencia de fortificaciones en ciudadelas pone en evidencia sus continuas disputas.

El período de Harappa se sitúa entre la mitad del tercer milenio y la mitad del segundo milenio a.C. El criterio más importante para fechar está constituido por los sellos indios encontrados en Mesopotamia (en Tell Asmar, Ur, etc.), asociados a la dinastía de Accad (2340-2200 a.C.).

La civilización del Indo, que recibe este nombre del gran sistema fluvial en el que se asentaron muchos de sus aldeas, poblados y ciudades,

4 Colección de himnos en honor de las divinidades compuesta por los sacerdote indoarios aproximadamente entre el 1500 y el 900 a.C. No es una narración de acontecimientos, pero incidentalmente proporciona datos de la vida de los arios. Es fiable desde el punto de vista histórico, ya que su composición es contemporánea del período que describe.

5 Ver VV.AA.: Manual de Historia Universal, Nájera, Madrid, 1983, Vol.ll, págs. 467-472.

6 Véase H. Kulke and D. Rothermund, A history of India, Routledge, London, 1995, págs. 24-26. 
ocupó una región mayor que el antiguo Egipto. Llamada también civilización o cultura de Harappa -como uno de los yacimientos más importantes-, tuvo una superficie más o menos triangular que ronda el millón trescientos mil kilómetros cuadrados. La mayoría de los 150 emplazamientos de esta cultura de la Edad del Bronce están situados en el actual Pakistán. Al parecer, los centros principales fueron los dos asentamientos de mayor tamaño: Harappa y Mohenjo-Daro.

Se piensa que esta extensa civilización urbana se desarrolló a partir de las anteriores culturas neolíticas de Afganistán y Baluchistán, al oeste. En el 4000 a.C. la agricultura estaba diversificada y, tanto en el valle del Indo como en la llanura de inundación, apareció una red de poblados y aldeas. Es probable que el posterior aumento de la población fomentara nuevos asentamientos en la región, y en el 3500 a.C., o incluso en fecha anterior, existían varias aldeas en el aluvión fértil.

A lo largo del milenio siguiente surgieron ciudades amuralladas, oficios especializados y redes comerciales. Alrededor de 2500 a.C., la etapa de formación - conocida como temprano período del Indo (y también como Harappa temprana o pre-Harappa)- desembocó en la madura civilización del Indo.

Entre el 2500 y el 1500 a.C. se amplia la vida urbana sobre centros más próximos al pie del Himalaya: por su yacimiento tipo se la llama Civilización del valle del Indo o de Harappa: Chanhu Daro Mohenjo-Daro, Harappa, Lothal, Rangpur, Rupar.

De los numerosos emplazamientos del indo que se conocen, por su tamaño mucho mayor da la sensación de que Mohenjo-Daro y Harappa eran las capitales. Ambos centros, cada uno de los cuales tenía una superficie de más de 100 hectáreas, y las ciudades más pequeñas —en su mayoría alrededor de 10 hectáreas - compartían una serie de características, lo que con toda probabilidad señala que fueron minuciosamente planificadas.

A la cultura de Harappa siguió la todavía más precaria cultura de Jhangar. Tiene la apariencia de que, al menos en un caso, son los últimos restos accesibles a un población del Indo que, huyendo de sus ciudades saqueadas y destruidas, intentó recuperar a pequeña escala, con medios precarios, y en zonas apartadas y de dificil acceso, su antiguo esplendor.

\section{CULTURAS URBANAS DEL INDO}

Como no es posible detenernos en la enumeración de todas y menos en la descripción de sus caracteres particulares, vamos a hacer una breve 
descripción de la que puede servirnos de prototipo para otras de su clase: Mohenjo-Daro, la ciudad a orillas del Indo, de planta regular y viviendas rectangulares, precursora en el tiempo de otras de su especie en el Oriente Cercano (Mesopotamia). Se ignora el papel jugó en la Antigüedad. Desconocemos incluso su antiguo nombre, pues el actual (Mohenjo-Daro significa "Ciudad de los Muertos») es muy posterior. Pero podemos afirmar que en su tiempo fue una gran ciudad.

Dentro de los límites de la ciudad encontramos un trazado urbano sistemático siguiendo, sin duda, un plan preconcebido. Tres grandes calles paralelas de 10 metros de anchura la cruzaban de norte a sur y dos de este a oeste formando barrios de 364 metros por 182 metros dentro de los cuales las calles principales se cruzaban en ángulo recto formando manzanas. Las secundarias eran estrechas llegando algunas a sólo 1,25 de anchura.

En la ciudad baja había diversas casas espaciosas y bien dotadas, pues disponían de retretes, agua de sus propios pozos, hogares para hacer fuego, a menudo también salas de baño. Las aguas residuales eran conducidas desde el patio, el piso superior y el tejado al exterior por medio de tuberías de barro ${ }^{7}$.

La relativa uniformidad de las habitaciones cabe atribuirla a la existencia de una clase media bastante numerosa de "burgueses comerciantes".

En este sector de la ciudad también se encontraban los talleres de diversos artesanos, como trabajadores del metal, ensartadores de cuentas, alfareros, albañiles y fabricantes de textiles. Dicho sea de paso, los paños de algodón hallados en Mohenjo-Daro y en Lothal son las primeras pruebas que existen sobre el cultivo del algodón. Se han encontrado muchas pesas de piedra, y es evidente que se contaba con un sistema normalizado de pesos y medidas. El sistema unitario de pesas se basaba en el sistema binario, usual en la India hasta los tiempos más recientes.

La mayoría de los edificios eran de adobes y ladrillos cocidos a fuego - lo que ha permitido la buena conservación hasta nuestros días del casco urbano- y en las calles había desagües cubiertos con ladrillos, y con sumideros para llevar a cabo inspecciones. En el extremo occidental de la

La ciudad estaba provista de ciertos servicios, como un sistema de conducción de agua que permitió tener no sólo fuentes públicas en abundancia, sino incluso suministro domiciliario, lo que, a su vez, posibilitó en las casas el uso del baño y hasta de retretes. Los desagües iban a dar a colectores, que corrían bajo el nivel de la calle, desembocaban en grandes cloacas, que discurrían bajo las vías principales. 
ciudad, se erigía una ciudadela (figura 3), levantada sobre una plataforma artificial de ladrillo y rodeada por fortificaciones de inmensa solidez, dentro de la cual había edificios públicos importantes: un gigantesco granero, un edificio, que probablemente era la sede de un colegio sacerdotal, una especie de sala de reuniones y baños. Fueron los excavadores los que pusieron nombre a estas estructuras, nombres sugeridos por su aspecto y por la posible función que desempeñaron.

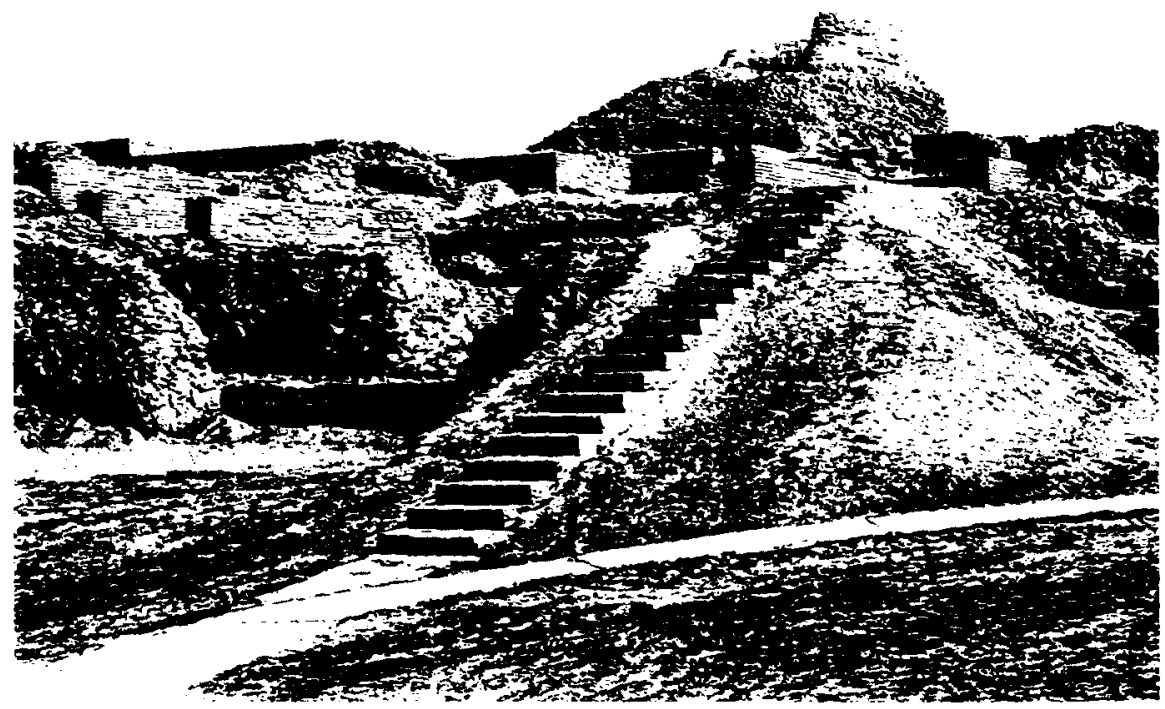

Figura 3. Ciudadela de Mohenjo-Daro.

El "gran baño» (figura 4), que posiblemente tuviera una función religiosa ${ }^{8}$, medía 55 por 33 metros. La zona central de baño tenía 12 metros de largo por 7 metros de ancho y fue impermeabilizada mediante una capa de betún, rellenada con arcilla y revestida de ladrillos cortados y quemados. Junto a la piscina habia un pórtico de veinte columnas.

La sociedad de Harappa y Mohenjo-Daro tenía un carácter esencialmente urbano; su economía de base muestra un elevado nivel de vida,

B Ciertas investigaciones han conducido a la idea de que tal vez el gran baño fue un centro de baños rituales vinculado a la vida religiosa de Mohenjo-Daro. Resulta tentador plantear la hipótesis de que desempeñó una función parecida a la de los grandes depósitos de agua bendita de los modernos templos hindúes, agua que se usa para purificaciones. En Benarés se cree que el agua es tan sagrada que puede purificar el alma y liberarla del ciclo del renacimiento definitivamente. 


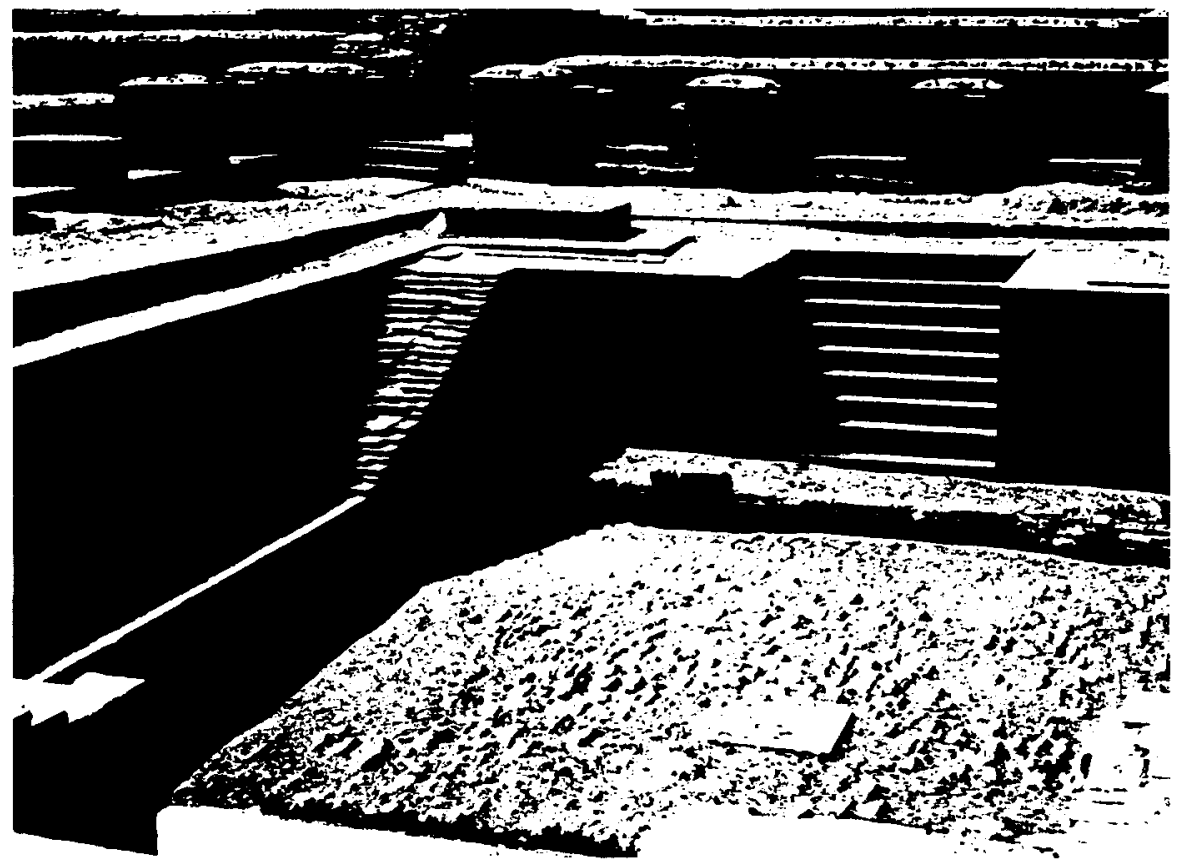

Figura 4. Instalaciones de los baños públicos en la antigua Mohenjo-Daro.

casi lujoso; dependía del comercio fluvial y la agricultura de las zonas de los alrededores. Su mejora estuvo en función de una técnica de irrigación artificial basada en un sistema de canales.

La base era la agricultura. Entre las especies que cultivaban destacan el trigo candeal, la cebada, el sésamo; es muy probable que se iniciase el cultivo del arroz. A su vez, adquire especial desarrollo el algodón ${ }^{9}$, que vino a ocupar el lugar del lino. Su ganadería disponía de un número elevado de animales domésticos, aunque se desconoce si entre ellos ya estaba el elefante. Las ciudades eran el centro del comercio, la manufactura y la industria, y abastecían con sus depósitos alimenticios

9 Se han encontrado en Mohenjo-Daro huellas de tejidos de algodón, las cuales evidencian que éste se cultivaba en la India en el 2000 a.C. por lo menos. A juzgar por el gran número de contrapesos de huso demasiado pequeños y ligeros para el hilado de la lana que se han hallado en las estaciones del valle del Indo, la tela de algodón tuvo que se localmente de uso común; que se manufacturaba también para la exportación queda probado por un trozo de tejido de algodón que, con una impresión de sello del tipo harappa, ha sido hallado en el Iraq. 
extensas regiones. Importaban de Afganistán, Rajastán, Irán o China: plomo, plata, cobre, lapislázuli, jade, quizá estaño, más algunas materias preciosas como la amazonita y el jade, utilizados para el lujo de las clases aristocráticas.

La cerámica harappa, toda ella hecha a la rueda, es en su mayor parte ordinaria, de producción en masa y puramente utilitaria, pero junto a estas vasijas encontramos vasos pulcramente pintados, por lo general en rojo y negro, pero a veces polícromos, con una decoración que tal vez tenga como primera fuente la anterior cerámica kulli, pero que, en la forma evolucionada en que se presenta en Harappa y Mohenjo-Daro, es propia de la cultura del valle.

Se ha dicho que los hindúes practican hoy una religión que se desarrolló principalmente durante los primeros mil años de la era actual. Sus raíces, sin embargo se encuentran 2500 años atrás, en la brillante civilización india que floreció en el valle del Indo aproximadamente entre el 2500 y 1500 a.C. Los pueblos arios que invadieron el noroeste de la India hacia el 1500 a.C. incorporaron algunas creencias y prácticas religiosas de los pueblos del valle del Indo a sus propias creencias; esto, transmitido por medio de sus antiguas escrituras, es clave en el hinduismo.

La prueba principal de que se dispone para poder reconstruir de alguna manera las prácticas religiosas de las gentes del Indo ${ }^{10}$, están limitadas a unas cuantas pequeñas figuras de piedra que tal vez representen deidades; figurillas de arcilla, algunas de las cuales eran meramente juguetes, y otras votivas, pero entre las que hay sin duda iconos religiosos; los muy numerosos sellos (figura 5) y amuletos entre los que algunos también ofrecen temas religiosos o figuras de dioses y, finalmente, piedras sagradas y símbolos fálicos.

Se desconocen las causas que pudieron motivar la desaparición de la cultura de Harappa. Los testimonios arqueológicos atestiguan que, mientras en el Indo central acaeció de manera violenta, en las zonas meridionales y orientales sobrevino de una forma gradual. El golpe mortal de Mohenjo-Daro fue dado por gentes arias que penetraron por el noroeste en torno al 1500 a.C., aunque la ciudad ya hacía mucho tiempo que agonizaba. Esta situación ha quedado recogida en los libros del Rig Veda.

10 Ver M.T. ROMAN, "La magia hindú y su proyección hacia occidente en el mundo antiguo", Espacio, Tiempo y Forma, UNED, Madrid, 1995, págs. 86-92. 


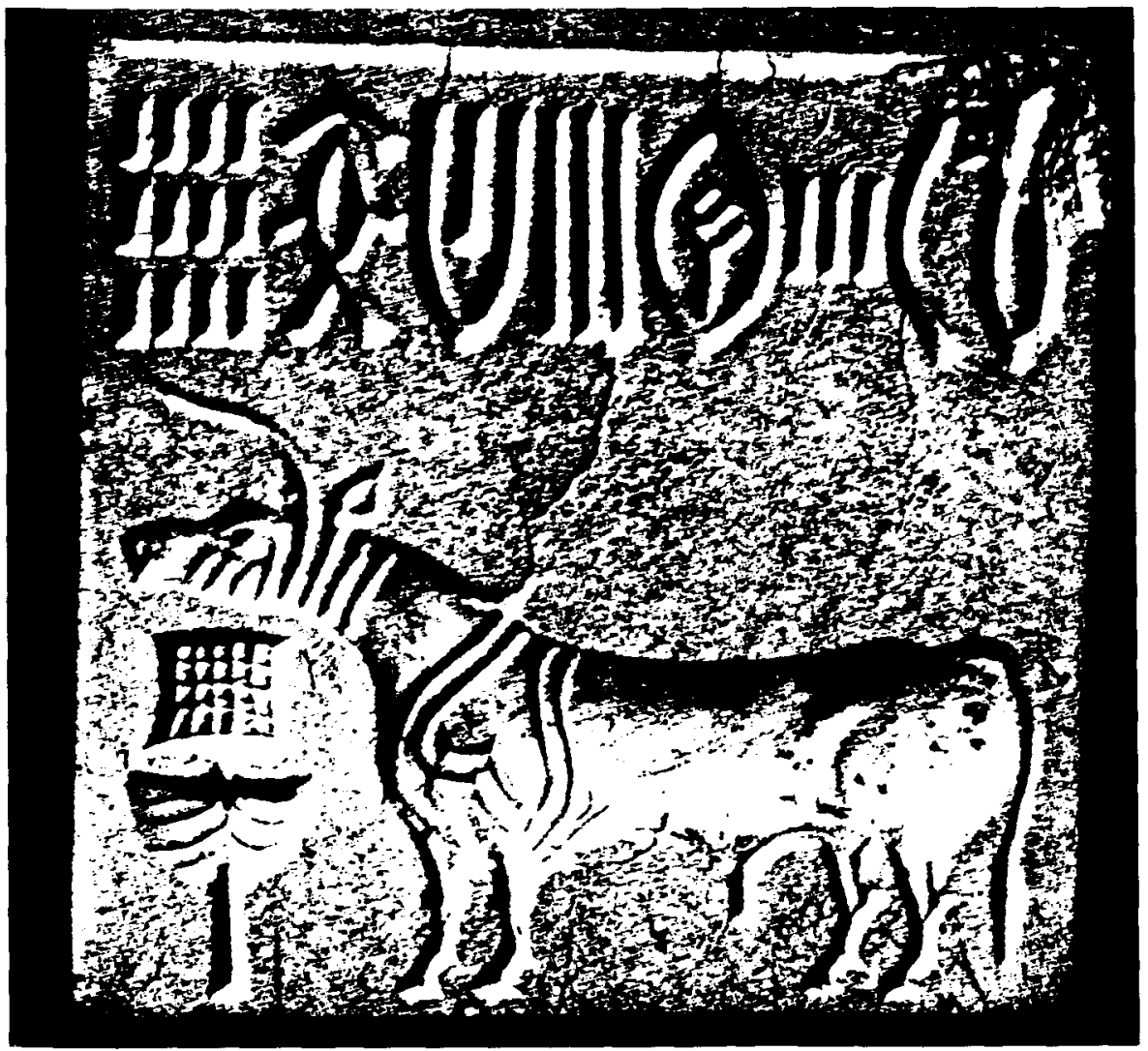

Figura 5. Sello con escritura pictográfica y unicornio de Harappa.

\section{BIBLIOGRAFÍA}

BOEKHOFF, H. y WINZER, F.: Historia de la cultura oriental, Labor, Madrid, 1968.

EMBREE, A.T. y WILHELM, F.: Historia del subcontinente desde las culturas del Indo hasta el comienzo del dominio inglés en Historia Universal, siglo XXI, Madrid, 1974.

GARcia, A.: Urbanística de las grandes ciudades del mundo antiguo, C.S.I.C., Instituto Español de Arquelogía, Madrid, 1885.

JAMES, E.O. (dir.): Historia de las religiones, Vergara, Barcelona, 1963, vol. 1.

Kulke, H. y Rothermund, D.: A history of India, Routledge, London, 1995.

Mann, G. y Heuss, A. (dirs.): Las culturas superiores de Asia Central y Oriental-2. Historia Universal. 11-2, Espasa-Calpe, Madrid, 1987.

Román, M.T.: Diccionario de las Religiones, Alderabán, Madrid, 1996.

- "La magia hindú y su proyección hacia occidente en el mundo antiguo", Espacio, Tiempo y Forma, UNED, Madrid, 1995.

VV.AA.: Manual de Historia Universal, Nájera, Madrid, 1983, vol. II.

WhEELER, S.M.: Indus Valley Civilization, Thames and Hudson, London, 1966 